\title{
Antisense-Oligonukleotide II
}

\section{Therapeutisches Potenzial bei neuropsychiatrischen Erkrankungen sowie ethische und gesundheitspolitische Aspekte}

\author{
H. Graf', D. Lehmann ${ }^{1,2}$, A. C. Ludolph², C. D. Wurster ${ }^{2}$ \\ ${ }^{1}$ Universitätsklinikum Ulm, Klinik für Psychiatrie und Psychotherapie III, Ulm; ${ }^{2}$ Universität- und Rehabilitationskliniken \\ Ulm, Neurologische Universitätsklinik, Ulm
}

\section{Schlüsselwörter \\ Antisense-Oligonukleotide, Alzheimer De- menz, psychische Störung}

\section{Zusammenfassung}

Antisense-Oligonukleotide (ASO) sind synthetische einzelsträngige Nukleinsäuren, die an die komplementäre Sequenz der prämRNA oder mRNA binden, Transkription und Translation beeinflussen und damit die Proteinsynthese modulieren. Im Jahr 2016 wurden 2 ASOs zur Behandlung neuromuskulärer Erkrankungen zugelassen. In tierexperimentellen sowie klinischen Studien zeigten sich vielversprechende Wirksamkeitsnachweise von ASOs zur Behandlung weiterer neurodegenerativer Erkrankungen. In diesem Artikel sollen die gegenwärtig zur Verfügung stehenden Ergebnisse zu ASOs bei der Behandlung der Alzheimer Demenz vorgestellt werden. Basierend auf in Tierexperimenten untersuchten ASOs sollen mögliche Zielstrukturen diskutiert werden, die zukünftig für einen Einsatz der ASO-Strategie in der Behandlung psychischer Erkrankungen sprechen könnten. Darüber hinaus sollen finanzielle und ethische Aspekte, die mit der Einführung von ASOs aufkamen, vorgestellt werden.

\section{Keywords}

Antisense oligonucleotides, Alzheimer disease, psychiatric disorders

\section{Summary}

Antisense oligonucleotides (ASO) are singlestranded nucleic acids strings that bind to a complementary sequence of the pre-mRNA or mRNA, modulate transcription and/or translation and thus, protein expression. In 2016, two ASOs were approved for treatment of neuromuscular disorders. Currently, there are further ASO drugs under research in animal models or even in clinical trials for the treatment of further neurodegenerative disorders with promising results. In this article, we present the results from studies conducted with ASO strategies for the treatment of Alzheimer's disease. Owing to previously investigated $\mathrm{ASO}$ s in experimental studies, we discuss potential targets of ASO strategies and whether this approach is eligible for treatment of psychiatric disorders. Moreover, we provide an overview of the upcoming discussion regarding costs, cost-effectiveness and ethical implications of ASOs.

Korrespondenzadresse

Priv.-Doz. Dr. med. Heiko Graf

Universitätsklinikum Ulm

Klinik für Psychiatrie und Psychotherapie III

Leimgrubenweg 12-14, $89073 \mathrm{Ulm}$

Tel. 0731/50061401, Fax 0731/50061402

heiko.graf@uni-ulm.de
Antisense oligonucleotides II: Treatment options in neuropsychiatric disorders, ethical and healthcare implications

Nervenheilkunde 2018; 37: 818-823

eingegangen am: 1. September 2018

angenommen am: 12. September 2018
Antisense-Oligonukleotide (ASO) sind synthetische, einzelsträngige Nukleinsäuren, die über Watson-Crick-Basenpaarung an die komplementäre Sequenz der prämRNA oder mRNA binden. In Abhängig- keit ihrer chemischen Struktur können ASO über verschiedene Wirkmechanismen Transkription und Translation und damit die Synthese krankheitsfördernder Proteine modulieren $(1,2)$. Neben der Genthera- pie steht damit eine Behandlungsstrategie zur Verfügung, die bereits in frühe Pathomechanismen eingreift.

Nach Jahrzehnten grundlagenwissenschaftlicher Untersuchungen konnten kürzlich ASOs in die klinische Praxis bei der Behandlung neurodegenerativer Erkrankungen eingeführt werden. Im Jahr 2016 wurde durch die amerikanische Arzneimittelbehörde FDA (United States Food and Drug Administration) das AntisenseOligonukleotid Eteplirsen für die Behandlung der Muskeldystrophie Duchenne zugelassen. Kurze Zeit später folgte die Zulassung eines weiteren Antisense-Oligonukleotids (Nusinersen) zur Behandlung der spinalen Muskelatrophie (SMA). Seit 2017 ist Nusinersen durch die Europäische Arzneimittelkommission (EMA) in Deutschland zur Behandlung der SMA zugelassen. Insbesondere in der Therapie der SMA konnten mit dem ASO Nusinersen beeindruckende Behandlungserfolge erzielt werden, die noch vor wenigen Jahren unvorstellbar waren. Darüber hinaus werden ASO-Strategien für andere neuromuskuläre oder neurodegenerative Erkrankungen wie der myotonen Dystrophie, der Chorea Huntington oder der Amyotrophen Lateralsklerose (ALS) in Tiermodellen oder in klinischen Studien an Patienten untersucht.

Die erfolgreiche Entwicklung der ASOTherapie und die vielversprechenden Ergebnisse in ihrer Anwendung bei neuromuskulären und neurodegenerativen Erkrankungen führen unweigerlich zur Frage, ob ASO-Strategien auch zur Behandlung psychischer oder neuropsychiatrischer Erkrankungen eingesetzt werden können.

Viele Gründe sprechen gegen den Einsatz von ASO-Strategien in neuro-/psychi- 
atrischen Erkrankungen. Der zugrunde liegende Pathomechanismus vieler psychischer Erkrankungen ist weitestgehend ungeklärt und Diagnosen werden im Wesentlichen auf der Grundlage der klinischen Beobachtung und der zur Verfügung stehenden Klassifikationssysteme gestellt. Erste Untersuchungen zur Genetik psychischer Störungen anhand von Zwillingsund Adoptionsstudien wurden Anfang und Mitte des 20. Jahrhunderts durchgeführt (3-5). Mittlerweile konnten durch molekulargenetische Untersuchungen und $\mathrm{Ge}$ nomstudien an einer großen Anzahl an Patienten, Hunderte von häufigen und seltenen genetischen Variationen bei neuropsychiatrischen Erkrankungen nachgewiesen werden (6). Die Hypothese „eine Genveränderung, eine Erkrankung", wie sie oft bei neuromuskulären Erkrankungen zu beobachten ist, gilt jedoch nach gegenwärtigem Kenntnisstand nicht bei psychischen Erkrankungen. Stattdessen basiert deren Ätiologie auf einer komplexen Interaktion zwischen Genen oder Genveränderungen, strukturellen Gehirnveränderungen, Gehirnentwicklung und Hirnreifungsprozessen, funktionellen Verbindungen zwischen Gehirnregionen, neurochemischen Veränderungen und Umweltfaktoren. Die Pathophysiologie psychischer Störungen ist demnach weniger gut charakterisiert als die von neuromuskulären oder neurodegenerativen Erkrankungen und Hypothesen zur Ätiologie sind oft noch nicht schlüssig oder basieren auf Epiphänomenen.

Andererseits waren die Fortschritte in der Behandlung neuromuskulärer und neurodegenerativer Erkrankungen durch ASOs noch vor wenigen Jahren ebenso wenig vorstellbar. Weiterhin konnten mittlerweile auch bei Erkrankungen, bei denen komplexe genetische Veränderungen eine Rolle spielen, ASO-Strategien erfolgsversprechend eingesetzt werden. Neben der ALS könnte die Alzheimer-Demenz (AD) ein weiteres Beispiel sein.

\section{ASOs bei AD}

Die AD ist die häufigste Ursache der Demenz und stellt eine der größten gesundheitspolitischen Herausforderungen des 21 . Jahrhunderts dar. Es wird vermutet, dass sich die Prävalenzraten alle 20 Jahre bis hin zum Jahr 2050 etwa verdoppeln (7). Klinische Symptome umfassen neben dem zunehmenden Verlust von Gedächtnisleistungen, eine verminderte Leistung der exekutiven Funktionen sowie eine Vielzahl psychischer Symptome, die die selbstständige Lebensführung der Betroffenen beeinträchtigen. Für die Erkrankung sind bislang vor allem 2 wesentliche molekulare Pathomechanismen bekannt:

- die extraneuronale Akkumulation und Ablagerung abnormal gefalteter Amyloidpeptide (A $\beta$ ) in Amyloid-Plaques,

- sowie intrazelluläre neurofibrilläre Aggregate und Ablagerungen von hyperphosphoryliertem tau-Protein in Form sogenannter ,tangles' $(8,9)$.

Monomeres $A \beta$ entsteht durch die proteolytische Spaltung des Amyloid-Vorläuferproteins APP (amyloid precursor protein), einem integralen Typ 1 Transmembranglykoprotein, von dem man annimmt, dass es für die Ausbildung von Synapsen eine Rolle spielt. Die proteolytische Spaltung von APP durch $\alpha$ - und $\gamma$-Sekretasen führt nicht zur Bildung von $A \beta$-Fragmenten bzw. zu deren Wiederaufnahme in endosomale Kompartimente (nicht amyloider Prozessierungsweg) (10). Die APP-Spaltung durch $\beta$ - und dann $\gamma$-Sektrasen führt hingegen dazu, dass das entstehende $A \beta$-Fragment zu unlöslichen Fibrillen und Plaques oligomerisiert (11). Dies wiederum führt über mehrere Mechanismen einschließlich der Aktivierung von Mikroglia zur Initiierung einer Immunantwort, oxidativem Stress, neurotoxischen Reaktionen und letztlich dem Untergang von Synapsen (amyloider Prozessierungsweg) (10).

Mittlerweile sind eine Reihe an Mutationen im APP-Gen bekannt, die zumeist pathogenetisch sind und zur autosomaldominant vererbten Form der AD mit frühem Beginn (familiäre Alzheimer-Krankheit) führen (10). Veränderungen des Proteins an den Schnittstellen der $\beta$ - und $\gamma$-Sektrasen führen dabei zur vermehrten Bildung von $A \beta$-Fragmenten oder zu einer veränderten Ratio an $A \beta_{1-40}: A \beta_{1-42}$ in Richtung der toxisch wirkenden $A \beta_{1-42}$-Peptide (12).

Im Jahr 2000 untersuchte eine Arbeitsgruppe im Tierexperiment (13) die Wir- kung eines Phosphorothioat modifizierten ASOs, das an die A-Region des APP-Gens bindet und zu einer verminderten Expression des C-terminals von APP, d. h. der Region aus der $A \beta$ entsteht, führt. Nach 2 intrazerebroventrikulären Injektionen konnten um 43-63\% geringere APP-Konzentrationen im Gehirn sowie eine Verbesserung von Gedächtnisleistungen und Lernen an Mäusen beobachtet werden. Diese Ergebnisse konnten im Tiermodell mit einem intravenös zu verabreichenden ASO mit ähnlicher Wirkung bestätigt werden (14). Die Nachteile dieser Untersuchungen lagen jedoch in der Verwendung spezifischer Tiermodelle, die die Übertragung dieser Ergebnisse auf den Menschen limitierten und die verwendeten ASOs überwanden nur in geringem Ausmaß die Blut-Hirn-Schranke; 2 wesentliche Umstände, die den Fortschritt in der Entwicklung von ASOs, die die APPGenexpression modulieren, verhinderten (11).

Genetische Veränderungen der $\mathrm{AD}$ sind nicht nur mit der frühen, sondern auch mit in höherem Alter und sporadisch auftretenden Formen der AD assoziiert. Eine der wichtigsten genetischen Risikofaktoren hierbei ist der Polymorphismus im APOEGen auf Chromosom 19q13.2. Das Gen kodiert für das am Lipidstoffwechsel beteiligte Apolipoprotein E (APOE) und weist einen Polymorphismus auf, aus dem 3 Isoformen des Lipoproteins entstehen. Die Apolipoprotein-Isoform ApoE- $\varepsilon 4$ ist neben weiteren Faktoren (15), mit einer vermehrten Ablagerung von $\mathrm{A} \beta$-Fragmenten assoziiert und geht mit einem 2- bis 12-fach erhöhten Risiko zur Entwicklung einer AD einher $(15,16)$.

Mittlerweile wurden mehrere Strategien zur Modulation der APOE-vermittelten Alzheimer-Pathologie einschließlich der adenovirusvektorgestützten Gentherapie im Tiermodell untersucht (15). Die wissenschaftlichen Untersuchungen zum Einsatz von ASO-Strategien konzentrierten sich jedoch auf die Modulation der Expression des Apolipoprotein-E2-Rezeptors (ApoER2), über den physiologische Langzeitpotenzierung (LTP), aber auch APOE-Effekte vermittelt werden. Für die Verstärkung von LTP und damit von Gedächtnisleistungen ist die Anwesenheit bestimmter Aminosäuren im ApoER2-Rezeptor erforderlich, 
die durch das Exon 18 (bei Mäusen Exon 19) des ApoER2-Gens kodiert werden. Dabei ist das akkurate Spleißen des Exons 18 von Bedeutung. So konnte ein veränderter Spleißprozess mit der Folge einer verminderten Inklusion von Exon 18 bei Patienten mit AD beobachtet werden (17) und kognitive Defizite korrelierten mit der verminderten Anzahl des Exons. Übereinstimmend hierzu zeigten Mäuse mit verändertem Spleißingmechanismus von Exon 19 eine verschlechterte Lernleistung. Hinrich und Kollegen (17) entwickelten am Tiermodell ein ASO, das an Exon 19 benachbarte Introns bindet und die Exklusion des Exons aus der RNA verhindert. Eine Einmalgabe des ASOs konnte den Spleißingmechanismus bis zu 6 Monate anhaltend modulieren und führte zu Verbesserungen von Lernen und Gedächtnis (17).

Ein weiterer wichtiger Pathomechanismus der $\mathrm{AD}$ und in anderen neurodegenerativen Erkrankungen ist die pathologische Ablagerung von tau-Protein. Das tau-Protein bindet physiologisch an Mikrotubuli, stabilisiert diese und ist an der intrazellulären Signaltransduktion und an der Neurogenese beteiligt (18). Im menschlichen $\mathrm{Ge}$ hirn werden typischerweise 6 Isoformen des tau-Proteins aus dem tau (MAPT)-Gen exprimiert, die sich aufgrund von alternativem Spleißen des tau-MAPT-Gens unterscheiden (11). Der Ausschluss von Exon 10 des MAPT-Gens führt dabei zur Expression eines tau-Proteins, das 3 Mikrotubulibindende Aminosäuresequenzwiederholungen enthält (3 MTBRs; 3R-tau Protein), während hingegen der Einschluss des Exon $10 \mathrm{zu} 4$ MTBRs im tau-Protein führt (4R-tau Protein) (19). Bei Gesunden liegt 3R- und 4R-tau-Protein im Verhältnis 1:1 vor, während eine Verschiebung der Ratio (4R > 3R-tau-Protein) bei mehreren Tauopathien nachgewiesen werden konnte (20).

Wenn man bedenkt, dass posttranslationale Modifikationen wie die tau-ProteinHyperphosphorylierung und die Akkumulation des Proteins wesentliche Faktoren in der Pathogenese der AD und des neuronalen Zelltodes darstellen $(9,19)$, so erscheint das Ziel die tau-Expression durch ASO zu reduzieren ein logischer Schritt zu sein.

Kürzlich wurden am Tiermodell die Effekte eines ASOs untersucht, das selektiv humane tau-mRNA supprimiert (21). Zu- sammenfassend konnten im Gehirn eine verminderte Konzentration an tau- und phosphoryliertem tau-Protein, eine verminderte Reduktion des Hippocampusvolumens, reduzierter neuronaler Zelltod und ein verlängertes Überleben im Tiermodell beobachtet werden. Eine alternative ASOStrategie fokussiert auf Mutationen im MAPT-Gen, die alternative Spleißprozesse von Exon 10 begünstigen. So konnte ein $2^{\prime}$-MOE phosphothioat modifiziertes ASO das Skipping von Exon 10 zugunsten der vorrangigen Expression von 3R-tau-Protein bewirken. Bemerkenswerterweise war es dadurch möglich, die Anzahl an 4R-tauProtein zu reduzieren, ohne dass der Einsatz des ASOs Auswirkungen auf die Gesamtkonzentration an tau-Protein hatte $(11,22)$.

Der Vorteil dieser ASO-Strategie liegt demnach in der Spezifität zur Reduktion von 4R-tau-Protein. Andererseits ließen sich ASOs, die die Gesamtkonzentration an tau-Protein vermindern, auch für andere Tauopathien z. B. der frontotemporalen Demenz (3R-Tauopathie) einsetzen.

Im Rahmen einer Phase-I/II-Studie wird ein ASO (ISIS 814907; IONIS MAPTRx; ClinicalTrials.gov. NCT03186989) an Patienten mit AD hinsichtlich der Arzneimittelsicherheit und der Nebenwirkungen untersucht. Das ASO bindet an die MAPT mRNA mit dem Ziel, die Gesamtkonzentration von tau-Protein unabhängig von der vorliegenden Isoform zu reduzieren. Im Tiermodell konnte nach intrathekaler Gabe des ASOs bereits eine 77\%ige Reduktion von MAPT-mRNA im frontalen Kortex und im Hippocampus (74\%ige Reduktion) nachgewiesen werden. Ergebnisse der humanen Phase-I/II-Studie stehe jedoch noch aus und werden mit Spannung erwartet.

\section{ASO-Strategie bei psychischen Störungen?}

Die Fortschritte in der Entwicklung von ASO-Strategien versprechen eine interessante Zukunft. ASOs, die für den Einsatz bei psychischen Störungen in Frage kämen, wurden in der Vergangenheit bereits tierexperimentell entwickelt bzw. untersucht.
Im Jahr 1978 wurde erfolgreich nachgewiesen, dass eine ASO-Strategie die Proteinexpression modulieren kann $(23,24)$. 1993 wurde eine ASO-Strategie im Tiermodell erstmals in vivo angewandt und so konzipiert, dass das ASO an die Neuropeptid Y1-Rezeptor-mRNA bindet und die Protein- bzw. Rezeptorexpression supprimiert.

Das Neuropeptid Y ist eines der am weitesten verbreiteten Neuropeptide im ZNS und bindet an G-Protein-gekoppelte Neuropeptid-Y-Rezeptoren, von denen 5 Subtypen (Y1, Y2, Y4-Y6) bekannt sind. NY1, NY2- und NY5-Rezeptoren werden am häufigsten im Hippocampus, in der Amygdala, im zingulären Kortex, im Thalamus und im Hypothalamus exprimiert und regulieren mitunter Lern- und Gedächtnisprozesse (25-27). Darüber hinaus ist NPY an der Modulation wichtiger biologischer Vorgänge wie der Regulation des Blutdrucks, der neuroendokrinen Sekretion, neuronalen Exzitabilität und Neuroplastizität beteiligt (28-33). Über NY1-Rezeptoren scheinen darüber hinaus Verhaltenseffekte wie die Stimulation von Nahrungsaufnahme (28), soziales Verhalten $(34,35)$, anxiolytische und antidepressive Effekte vermittelt zu werden $(36,37)$.

Die wiederholte intraventrikuläre Injektion des ASO führte zu einer signifikanten und spezifischen Reduktion der NY1-Rezeptorexpression im Gehirn von Ratten (38). Durch die verminderte Verfügbarkeit dieser Rezeptoren waren offenbar auch die NY1-vermittelten Effekte aufgehoben und die Tiere zeigten signifikant mehr ängstliches Verhalten. Durch die Gabe eines anderen ASOs, das nicht die Expression des NY1-Rezeptors, sondern die des Proteins (Neuropeptid Y) selbst supprimierte, konnte eine verminderte Nahrungsaufnahme induziert werden (39). Die genannten Studien waren zur damaligen Zeit nicht nur ein Beleg dafür, dass ASO-Strategien in vivo im ZNS angewandt werden können. Sie geben genauso einen Hinweis darauf, dass durch ASOs Verhalten und psychopathologische Phänomene moduliert werden könnten.

Der Wachstumsfaktor BDNF (brain derived neurotrophic factor) wäre eine weitere potentielle Zielstruktur für den möglichen Einsatz von ASO-Strategien. Die Rol- 
le des BNDF in der Ätiologie der depressiven Störung wird seit langem diskutiert (40), darüber hinaus ist der Wachstumsfaktor jedoch in entscheidendem Maße an der Formation von Synapsen und der Modulation von Neuroplastizität und Kognition beteiligt $(40,41)$. Die Möglichkeit der Modulation von BDNF-Expression mittels ASOs konnte bereits demonstriert werden. Ein ASO wurde im Mausmodell derart konzipiert, dass es an das ,Natural-antisense ${ }^{c}$-Transkript (NAT) bindet und inhibiert. Hierdurch kam es gewissermaßen zur Aufhebung der Inhibition und letztlich zu einer 2- bis 7-fachen Erhöhung der BDNFmRNA und der BDNF-Proteinkonzentration, einhergehend mit einem vermehrten Neuronenwachstum in vitro und in vivo (42). Weiterhin wäre die Modulation von Dopaminrezeptoren durch ASO-Strategien vorstellbar. Im Jahr 1993 konnte an Ratten gezeigt werden, dass ein ASO, das Dopamin-D2-Rezeptor (DR2) mRNA inhibiert, zu einer signifikanten Reduktion striataler DR2-Expression führt (43). Im Jahr 2002 konnten diese Befunde nochmals bestätigt werden. Nach dreimaliger Injektion eines ASO, das komplementär an die DR2-mRNA band und die Transkription inhibiert, reduzierte sich 6,12 und 24 Stunden nach der letzten Gabe signifikant die DR2-Anzahl bei unveränderter Konzentration an D1-, D2-, D4- und D5-Rezeptoren (44). Die Effekte einer antipsychotisch wirksamen Medikation beruhen mitunter auf der Blockade von D2-Rezeptoren. Allerdings wird nach längerer Einnahme von Antipsychotika auch eine vermehrte Rezeptorexpression (DR2-upregulation) beobachtet, die vermutlich für die Entwicklung motorischer Nebenwirkungen wie tardive Dyskinesien mitverantwortlich ist (45). Der Einsatz von ASOs mit dem Ziel die DR2-Rezeptorexpression zu vermindern, wäre demnach eine Möglichkeit, den DR2-Rezeptor-vermittelten Effekten und Nebenwirkungen entgegenzuwirken.

Wir sind uns jedoch darüber bewusst, dass die in diesem Abschnitt vorgestellten Strategien gegenwärtig (noch) theoretisch und einige Hürden bis zum Einsatz von ASOs in der Behandlung psychischer Störungen $\mathrm{zu}$ überwinden sind. Um ASOStrategien in der Psychiatrie anwenden zu können, bedarf es einer besseren Aufklärung der Ätiopathogenese und der erheblichen Verbesserung diagnostischer Maßnahmen. Eine große Herausforderung liegt darin, molekulare und zelluläre Veränderungen aufzudecken und diese dann auf genetische Veränderungen (Genotyp) zurückzuführen. Darüber hinaus müssen in der Pathogenese psychischer Störungen weiterhin molekulare, zelluläre und biochemische Veränderungen identifiziert und wiederum einzelnen psychiatrischen Erkrankungsbilder zugeordnet werden (molekulare Pathologie) (46). Obwohl die zur Verfügung stehenden ASOs ein gutes Nebenwirkungsprofil aufweisen $(47,48)$, gibt es keine Erfahrung in der längerfristigen Anwendung bei Hirnerkrankungen. Weiterhin sind die Effekte einer beispielsweise längerfristigen Erhöhung von einzelnen Zielproteinen weitestgehend unbekannt. Es konnte jedoch im Tiermodell gezeigt werden, dass $z$. B. die längerfristige Überexpression von BDNF wiederum mit schlechteren Lernleistungen und Gedächtnisfunktionen einherging (49).

Voraussichtlich werden noch Jahre oder Jahrzehnte vergehen bis ASOs in der Behandlung psychischer Störungen eingesetzt werden können. Die Aussicht auf eine Behandlungsmöglichkeit, die bereits auf Ebene der Transkription und Translation eingreift, sollte jedoch Ansporn sein, um die grundlagenwissenschaftliche und tierexperimentelle Untersuchung psychischer Störungen, die dringend erforderlich ist, weiter voranzutreiben.

\section{Ethische und finanzielle Aspekte der ASO-Strategie}

Gleichzeitig zum Enthusiasmus über den erfolgreichen Einsatz von ASOs und zur Zulassung zweier ASOs in der Behandlung neuromuskulärer Erkrankungen, kam eine andauernde und wichtige ethische und finanzielle Diskussion auf. Durch die Behandlung der Muskeldystrophie Duchenne mit dem ASO Eteplirsen fallen monatlich etwa 57600 USD Behandlungskosten an, obwohl Zweifel geäußert werden, ob der geringfügige Konzentrationsanstieg von 23\% an Dystrophin in der Muskulatur einer klinisch bedeutsamen Progression der Erkrankung entgegenwirken kann (50, 51). Für das ASO Nusinersen zur Behandlung der SMA liegen die Kosten noch höher. Nusinersen ist eines der teuersten zugelassenen Medikamente weltweit und die Kosten belaufen sich pro Patient allein für das Medikament auf über 500000 Euro im ersten Jahr, und in jedem weiteren Jahr auf jährlich 250000 Euro. Diese Preise sind von keinem Gesundheitssystem unter Berücksichtigung der Entwicklung weiterer ASOs langfristig zu bewältigen. So wurde für Nusinersen die Kostenübernahme durch die Krankenkassen in einigen Ländern auf die Behandlung von SMA Typ 1 Patienten eingegrenzt (52) oder gänzlich abgelehnt (51). Langfristige Wirksamkeitsnachweise von ASOs fehlen bei der Behandlung neuromuskulärer oder neurodegenerativer Erkrankungen. Die Evaluation der klinischen Wirksamkeit nach 1 oder 2 Jahren wäre nicht nur unter Berücksichtigung einer finanziellen Verantwortung, sondern auch der einer invasiven Applikation, nur eine von mehreren Möglichkeiten. Aus nachvollziehbaren Gründen initiierte die Fachzeitschrift Lancet im Jahr 2017 eine noch anhaltende Diskussion zu Fairness, dem Anstieg von Arzneimittelkosten, insbesondere bei seltenen Erkrankungen und den Verfahren zur Arzneimittelzulassung (50-53). Es ist verständlich, dass Betroffenen eine wirksame Behandlungsmöglichkeit unabhängig der Kosten nicht vorenthalten werden soll und kann. Weiterhin sind die hohen Arzneimittelkosten der Pharmakonzerne unter Berücksichtigung der Entwicklungskosten nachvollziehbar. Andererseits bringen die Kosten eine ethische Diskussion mit sich und es stellt sich die Frage, wie der Zugang zu diesen Arzneimitteln in Ländern mit einem weniger finanzkräftigen Gesundheitssystem gewährleistet werden kann. Diese kontroversen Fragen gilt es zu lösen und sie bedürfen nicht nur der engen $\mathrm{Zu}$ sammenarbeit zwischen Patientenvertretern, Ärzten, Wissenschaftlern, Krankenversicherungen, Pharmakonzernen und Gesundheitspolitik, sondern auch die einer gesamtgesellschaftlichen Diskussion. 


\section{Fazit für die klinische Praxis}

Mit dem Fortschritt in der Entwicklung von ASOs stehen erstmals Behandlungsoptionen zur Verfügung, die den Verlauf neurodegenerativer Erkrankungen modulieren können. Die erfolgsversprechenden Ergebnisse aus tierexperimentellen und klinischen Studien führen zu der Frage, ob ASO-Strategien auch zur Behandlung psychischer Störungen zukünftig eingesetzt werden könnten. Die komplexe, multigenetische und multikausale Ätiopathogenese psychischer Störungen spricht zunächst dagegen. Allerdings können bei neurodegenerativen oder neuropsychiatrischen Erkrankungen multifaktorieller Genese wie der Amyotrophen Lateralsklerose und der Alzheimer-Demenz, ASO-Strategien erfolgreich entwickelt und angewandt werden. Die detaillierte Aufklärung des Genotyps und der molekularen Pathologie psychischer Störungen ist eine zwingende Voraussetzung für die Entwicklung von ASO-Strategien in der Psychiatrie und eine Hürde, die erst überschritten werden muss. Die Aussicht auf eine Behandlungsoption kann jedoch ein weiterer Ansporn zu molekulargenetischen Untersuchungen psychischer Störungen sein, die ohne Zweifel erforderlich sind. Obwohl in bisherigen Untersuchungen keine schwerwiegenden unerwünschten Arzneimittelwirkungen zu beobachten waren, ist noch wenig bekannt über Langzeit- oder „Off-target"-Effekte. Darüber hinaus erfordert die Einführung von ASOs unter Berücksichtigung der hohen Arzneimittelkosten dieser Präparate, nicht nur eine Diskussion der finanziellen, sondern auch der damit verbundenen ethischen Aspekte, und kann nur unter Berücksichtigung der Meinungen aller Beteiligten einschließlich der Vertreter von Patienten, Ärzten, Wissenschaftler, Krankenversicherungen, Pharmakonzernen und der Gesundheitspolitik geführt werden.

\section{Interessenkonflikt}

Heiko Graf: keine Interessenkonflikte. Diana Lehmann: keine Interessenkonflikte. Claudia Diana Wurster: Beratertätigkeit für die Firma Hoffmann-La Roche; Honorarvorträge für die Firma Biogen sowie
Teilnahme an einem AdBoard Meeting der Firma Biogen. Albert Ludolph: Unterstützung für klinische Forschungsprojekte durch AB Science, Biogen Idec, Cytokinetics, GSK, Orion Pharam, Novartis, TauRx Therapeutics Ltd. und TEVA Pharmaceuticals. Honorare als Berater von Mitsubishi, Orion Pharma, Novartis, Teva sowie für lectures fees von Biogen und Ionis sowie als AdBoard-Mitglied von Biogen, Treeway, Hoffmann-La Roche und Novartis.

\section{Literatur}

1. Evers MM, Toonen LJA, van Roon-Mom WMC. Antisense oligonucleotides in therapy for neurodegenerative disorders. Adv Drug Deliv Rev 2015; 87: 90-103.

2. Bennett CF, Baker BF, Pham N, Swayze E, Geary RS. Pharmacology of Antisense Drugs. Annu Rev Pharmacol Toxicol 2017; 57: 81-105.

3. Kendler KS, Zerbin-Rüdin E. Abstract and Review of „Studien Über Vererbung und Entstehung Geistiger Störungen. I. Zur Vererbung und Neuentstehung der Dementia praecox." (Studies on the Inheritance and Origin of Mental Illness: I. To the Problem of the Inheritance and Primary Origin of Dementia Praecox.). Am J Med Genet - Semin Med Genet 1996; 67: 338-342

4. Luxenburger H. Vorläufiger Bericht über psychiatrische Serienuntersuchungen an Zwillingen. Zeitschrift für die gesamte Neurol und Psychiatr 1928; 116: 297-326.

5. Heston LL. Psychiatric disorders in foster home reared children of schizophrenic mothers. $\mathrm{Br} \mathrm{J}$ Psychiatry 1966; 112: 819-825.

6. Smoller JW, Andreassen OA, Edenberg HJ, Faraone S V, Glatt SJ, Kendler KS. Psychiatric genetics and the structure of psychopathology. Mol Psychiatry 2018; 4634: 1-13.

7. Prince M, Bryce R, Albanese E, Wimo A, Ribeiro W, Ferri CP. The global prevalence of dementia: A systematic review and metaanalysis. Alzheimer's Dement 2013; 9: 63-75.

8. Scheltens $\mathrm{P}$, Blennow $\mathrm{K}$, Breteler MMB, de Strooper B, Frisoni GB, Salloway S, Van der Flier WM. Alzheimer's disease. Lancet 2016; 388: 505-517.

9. Braak H, Tredici KD. Neuroanatomy and Pathology of Sporadic Alzheimer's Disease. New York: Springer 2015.

10. O’Brien RJ, Wong PC. Amyloid Precursor Protein Processing and Alzheimer's Disease. Annu Rev Neurosci 2011; 34: 185-204.

11. Schoch KM, Miller TM. Antisense Oligonucleotides: Translation from Mouse Models to Human Neurodegenerative Diseases. Neuron 2017; 94: 1056-1070.

12. St George-Hyslop PH. Molecular genetics of Alzheimer's disease. Biol Psychiatry 2000; 47: 183-199.

13. Kumar VB, Farr SA, Flood JF, Kamlesh V, Franko M, Banks WA, Morley JE. Site-directed antisense oligonucleotide decreases the expression of amyloid precursor protein and reverses deficits in learning and memory in aged SAMP8 mice. Peptides 2000; 21: 1769-1775.

14. Farr SA, Erickson MA, Niehoff ML, Banks WA, Morley JE. Central and peripheral administration of antisense oligonucleotide targeting amyloid- $\beta$ protein precursor improves learning and memory and reduces neuroinflammatory cytokines in Tg2576 (AßPPswe) mice. J Alzheimer's Dis 2014; 40: 1005-1016.

15. Yamazaki Y, Painter MM, Bu G, Kanekiyo T. Apolipoprotein E as a Therapeutic Target in Alzheimer's Disease: A Review of Basic Research and Clinical Evidence. CNS Drugs 2016; 30: 773-789.

16. Corder E, Saunders A, Strittmatter W, Schmechel D, Gaskell P, Small G, Roses A, Haines J, PericakVance M. Gene dose of apolipoprotein E type 4 allele and the risk of Alzheimer's disease in late onset families. Science 1993; 261: 921-923.

17. Hinrich AJ, Jodelka FM, Chang JL, Brutman D, Bruno AM, Briggs CA, James BD, Stutzmann GE, Bennett DA, Miller SA, Rigo F, Marr RA, Hastings ML. Therapeutic correction of ApoER2 splicing in Alzheimer's disease mice using antisense oligonucleotides. EMBO Mol Med 2016; 8: 328-345.

18. Morris M, Maeda S, Vossel K, Mucke L. The Many Faces of Tau. Neuron 2011; 70: 410-426.

19. Kolarova M, García-Sierra F, Bartos A, Ricny J, Ripova D. Structure and pathology of tau protein in Alzheimer disease. Int J Alzheimers Dis 2012; 201: 731526 .

20. Liu F, Gong C-X. Tau exon 10 alternative splicing and tauopathies. Mol Neurodegener 2008; 3: 8 .

21. DeVos SL, Miller RL, Schoch KM, Holmes BB, Kebodeaux CS, Wegener AJ, Chen G, Shen T, Tran H, Nichols B, Zanardi TA, Kordasiewicz HB, Swayze EE, Bennett CF, Diamond MI, Miller TM. Tau reduction prevents neuronal loss and reverses pathological tau deposition and seeding in mice with tauopathy. Sci Transl Med 2017; 9: 1-14.

22. Schoch KMM, DeVos SLL, Miller RLL, Chun SJJ, Norrbom M, Wozniak DFF, Dawson HNN, Bennett CF, Rigo F, Miller TMM. Increased 4R-Tau Induces Pathological Changes in a Human-Tau Mouse Model. Neuron 2016; 90: 941-947.

23. Zamecnik PC, Stephenson ML. Inhibition of Rous sarcoma virus replication and cell transformation by a specific oligodeoxynucleotide. Proc Natl Acad Sci USA 1978; 75: 280-284.

24. Stephenson ML, Zamecnik PC. Inhibition of Rous sarcoma viral RNA translation by a specific oligodeoxyribonucleotide. Proc Natl Acad Sci 1978; 75: 285-288.

25. Herzog H. Regional distribution of Y-receptor subtype mRNAs in rat brain. Eur J Neurosci 1999; 11: 1431-1448

26. Kornhuber J, Zoicas I. Neuropeptide Y prolongs non-social memory and differentially affects acquisition, consolidation, and retrieval of non-social and social memory in male mice. Sci Rep 2017; 7: 6821 .

27. Blomqvist AG, Herzog H. Y-receptor subtypes how many more? Trends Neurosci 1997; 20: 294-298.

28. Stanley BG, Leibowitz SF. Neuroreptide Y: Stimulation of feeding and drinking by injection into 
the paraventricular nucleus. Life Sci 1984; 35: 2635-2642.

29. Colmers WF, Bleakman D. Effects of neuropeptide $\mathrm{Y}$ on the electrical properties of neurons. Trends Neurosci 1994; 17: 373-379.

30. Vezzani A, Sperk G, Colmers WF. Neuropeptide Y: emerging evidence for a functional role in seizure modulation. Trends Neurosci 1999; 22: 25-30.

31. Michalkiewicz M, Michalkiewicz T, Kreulen DL, McDougall SJ. Increased blood pressure responses in neuropeptide $\mathrm{Y}$ transgenic rats. Am J Physiol Regul Integr Comp Physiol 2001; 281: 417-426.

32. Magni P. Hormonal control of the neuropeptide $Y$ system. Curr Protein Pept Sci 2003; 4: 45-57.

33. Hökfelt T, Stanic D, Sanford SD, Gatlin JC, Nilsson I, Paratcha G, Ledda F, Fetissov S, Lindfors C, Herzog H, Johansen JE, Ubink R, Pfenninger KH. NPY and its involvement in axon guidance, neurogenesis, and feeding. Nutrition 2008; 24: 860-868.

34. Sajdyk TJ, Vandergriff MG, Gehlert DR. Amygdalar neuropeptide Y Y1 receptors mediate the anxiolytic-like actions of neuropeptide $\mathrm{Y}$ in the social interaction test. Eur J Pharmacol 1999; 368: 143-147.

35. Sajdyk TJ, Schober DA, Smiley DL, Gehlert DR. Neuropeptide Y-Y2 receptors mediate anxiety in the amygdala. Pharmacol Biochem Behav 2002; 71: 419-423.

36. Redrobe JP, Dumont Y, Fournier A, Quirion R. The neuropeptide Y (NPY) Y1 receptor subtype mediates NPY-induced antidepressant-like activity in the mouse forced swimming test. Neuropsychopharmacology 2002; 26: 615-624.

37. Enman NM, Sabban EL, McGonigle P, Van Bockstaele EJ. Targeting the neuropeptide $\mathrm{Y}$ system in stress-related psychiatric disorders. Neurobiol Stress 2015; 1: 33-43.

38. Wahlestedt C, Pich EM, Koob GF, Yee F, Heilig M. Modulation of anxiety and neuropeptide Y-Y1 receptors by antisense oligodeoxynucleotides. Science 1993; 259: 528-531.

39. Akabayashi A, Wahlestedt C, Alexander JT, Leibowitz SF. Specific inhibition of endogenous neuropeptide Y synthesis in arcuate nucleus by antisense oligonucleotides suppresses feeding behavior and insulin secretion. Mol Brain Res 1994; 21: 55-61.

40. Castren E, Voikar V, Rantamaki T. Role of neurotrophic factors in depression. Curr Opin Pharmacol 2007; 7: 18-21.

41. Castren E, Antila H. Neuronal plasticity and neurotrophic factors in drug responses. Mol Psychiatry 2017; 22: 1085-1095.

42. Modarresi F, Faghihi MA, Lopez-Toledano MA, Fatemi RP, Magistri M, Brothers SP, Van Der Brug MP, Wahlestedt C. Inhibition of natural antisense transcripts in vivo results in gene-specific transcriptional upregulation. Nat Biotechnol 2012; 30: 453-459.

43. Zhang M, Creese I. Antisense oligodeoxynucleotide reduces brain dopamine D2 receptors: behavioral correlates. Neurosci Lett 1993; 161: 223-226.

44. Andoh H, Yoshikawa M, Kawaguchi M, Matsumoto $\mathrm{H}$, Yamazaki K, Oka T. The selective action of D2 dopamine receptor antisense oligodeoxynucleotide on the expression of the dopamine receptor subtype mRNA in rat striatum. Tokai J Exp Clin Med 2006; 31: 73-77.

45. Silvestri S, Seeman M V., Negrete JC, Houle S, Shammi CM, Remington GJ, Kapur S, Zipursky RB,
Wilson AA, Christensen BK, Seeman P. Increased dopamine D2receptor binding after long-term treatment with antipsychotics in humans: A clinical PET study. Psychopharmacology 2000; 152 $174-180$

46. McCammon JM, Sive H. Challenges in understanding psychiatric disorders and developing therapeutics: a role for zebrafish. Dis Model Mech 2015; 8: 647-656.

47. Schoch KM, Miller TM. Antisense Oligonucleotides: Translation from Mouse Models to Human Neurodegenerative Diseases. Neuron 2017; 94: 1056-1070.

48. Khorkova O, Wahlestedt C. Oligonucleotide therapies for disorders of the nervous system. Nat Biotechnol 2017; 35: 249-263

49. Cunha C, Angelucci A, D’Antoni A, Dobrossy MD, Dunnett SB, Berardi N, Brambilla R. Brain-derived neurotrophic factor (BDNF) overexpression in the forebrain results in learning and memory impairments. Neurobiol Dis 2009; 33: 358-368.

50. Dalakas MC. Gene therapy for Duchenne muscular dystrophy: Balancing good science, marginal efficacy, high emotions and excessive cost. Ther Adv Neurol Disord 2017; 10: 293-296.

51. Gellad WF, Kesselheim AS. Accelerated Approval and Expensive Drugs - A Challenging Combination. N Engl J Med 2017; 376: 2001-2004.

52. The Lancet Neurology. Treating rare disorders: time to act on unfair prices. Lancet Neurol 2017; 16: 761.

53. Prasad V. Nusinersen for spinal muscular atrophy are we paying too much for too little? JAMA Pediatr 2018; 172: 123-125. 


\section{Zertifizierte Fortbildung}

Fragen zum Thema „Antisense-Oligonukleotide I: Chemische Modifikationen, Wirkmechanismen und therapeutischer Einsatz bei neurodegenerativen Erkrankungen“ und „Antisense-Oligonukleotide II: Therapeutisches Potenzial bei neuropsychiatrischen Erkrankungen sowie ethische und gesundheitspolitische Aspekte"

1. Welche Aussage zum Wirkmechanismus von Antisense-Oligonukleotiden (ASO) ist richtig?

a) Im Jahre 1988 wurde erstmals gezeigt, dass ASO die Adenosintriphosphatregeneration in den Mitochondrien steigern kann.

b) ASO stabilisiert das Zytoskelett durch Vermehrung der Aktinfilamente und Verlängerung der Mikrotubuli.

c) Durch Bindung an die m-RNA können ASO sehr spezifisch die Biosynthese einzelner Proteine reduzieren.

d) Während der initialen Interphase der Mitose binden ASO am Zentromer und modifizieren dabei die DNA.

e) ASO docken wie Transmitter an Ribosomen an und fördern so spezifisch die Proteinbiosynthese.

2. Welche Aussage zu den notwendigen chemischen Modifikationen von ASO ist falsch?

a) Die Demethylierung der 2'-0 Gruppe des Riboserings verstärkt die Affinität zur ZielRNA.

b) Chimerische ASOs werden von modifizierten Oligonukleotiden an den Enden des Phosphtoiat-Grundgerüstes flankiert.

c) Hepatotoxizität wird durch die Kombination mit einer Methoxyethylierung am Ribosering reduziert.

d) Die Ausbildung eines zyklischen Ethers zwischen der 2'-OH-Gruppe und des Riboseringes führt zu einer erhöhten Bindungsaffinität der ASOs.

e) Die Nukleasenresistenz wird durch eine 0 $\rightarrow$ S-Substitution in der Phosphodiestergruppe verbessert.

3. Welche Aussage zur Behandlung der spinalen Muskelatrophie (SMA) mit dem ASO Nusinersen ist richtig?

a) Die Behandlung mit dem ASO Nusinersen ist ausschließlich bei SMA Typ 3 zugelassen.

b) Nusinersen führt dazu, dass die Funktion des SMN2-Gens „ausgeschaltet” wird.

c) Nusinersen muss intrathekal verabreicht werden. d) Die Behandlung der SMA mit Nusinersen ist in Deutschland noch nicht zugelassen.

e) Die Behandlung mit Nusinersen führt zu erhöhten Konzentrationen des Strukturproteins Dystrophin.

\section{Welche Aussage zur Muskeldystrophie} Duchenne ist richtig?

a) Bei der Mehrzahl der Dystrophin-Mutationen handelt es sich um Punktmutationen.

b) Durch den umschriebenen und spezifischen Pathomechanismus ist eine ASO-Strategie ausreichend, um das gesamte Spektrum der Erkrankung zu behandeln.

c) Durch die Anwendung des ASOs Drisapersen zeigte sich auch nach 49 Wochen noch eine anhaltende signifikante Verbesserung der Gehstrecke.

d) Das Exon-Skipping bildet die Grundlage vieler ASO-Strategien.

e) Seit September 2016 ist das ASO Drisapersen zur Behandlung der Muskeldystrophie Duchenne zugelassen.

\section{Welche Aussage zur Myotonen Dystro-} phie (MD) ist falsch?

a) Ein ASO zur Behandlung der Myotonen Dystrophie befindet sich bereits in klinischen Studien.

b) Der Myotonen Dystrophie Typ 1 und Typ 2 liegt ein Gendefekt mit Tri- bzw. Tetranukleotid-Expansionen zugrunde.

c) Myotone Dystrophien sind autosomal-dominante Multisystemerkrankungen.

d) Eteplirsen wurde im September 2016 zur Behandlung der Myotonen Dystrophie zugelassen.

e) Das Ziel einer ASO-Strategie bei Myotoner Dystrophie könnte die Reduktion der RNAExpression des DMPK1-Gens sein.

6. Welche Aussage zur Amyotrophen Lateralsklerose (ALS) ist richtig?

a) Bei etwa $45 \%$ der ALS Fälle findet sich eine spezifische Vererbungskomponente.

b) Target für eine ASO-Therapie der ALS könnten sowohl das SOD1-Gen als auch die C9orf72 Repeat-Expansion sein.
CME-Fortbildung online

Die Teilnahme an dieser durch die Ärztekammer Nordrhein zertifizierten CME-Fortbildung ist für 12 Monate ausschließlich online möglich. Zur Anmeldung gehen Sie bitte auf http://cme.thieme.de. Dort erfahren Sie auch den genauen Teilnahmeschluss. Es ist immer nur eine Antwort pro Frage zutreffend. Als Leser der Nervenheilkunde nehmen Sie kostenlos am CME-Programm teil. Je nach CME-Fortbildung erhalten Sie bis zu vier Fortbildungspunkte. Weitere Informationen zur Anmeldung und Registrierung finden Sie unter http://cme.thie me.de. Sie erhalten bei inhaltlichen und technischen Fragen tutoriellen Support. VNR 2760512018154652846

c) Die Genmutationen bei sporadischen und bei vererbten ALS-Fällen unterscheiden sich grundsätzlich.

d) Bisher wurden ASOs zur Behandlung der ALS nur im Tierexperiment und noch nicht am Patienten untersucht.

e) Das am Exon 51 der Dystrophin prä-mRNA bindende ASO ist zur Therapie der ALS in den USA zugelassen.

\section{Welche Aussage zu Chorea Huntington} ist falsch?

a) Der Erkrankung liegt pathogenetisch eine Triknukleotid-Amplifikation zugrunde.

b) Ab 40 CAG-Wiederholungen ist mit einer vollständigen Penetranz der Erkrankung zu rechnen.

c) Eine mögliche ASO-Strategie bewirkt einen enzymatisch vermittelten Abbau der HTTmRNA über RNase $\mathrm{H} 1$.

d) Das ASO IONIS-HTTRx befindet sich bereits in klinischen Studien.

e) Im Tiermodell konnte eine Verminderung der mutierten HTT-mRNA um lediglich 10\% nachgewiesen werden.

\section{Welche Aussage zur Alzheimer-Demenz} ist falsch?

a) Das ASO IONIS-MAPTRx zur Behandlung der Alzheimer-Demenz befindet sich bereits in einer klinischen Studienphase.

b) Im Tiermodell führte das ASO IONISMAPTRx zu einer über 70\%igen Reduktion der MAPT-mRNA im Hippocampus.

c) Bei der Alzheimer-Demenz liegt eine Verschiebung der Ratio (3R- > 4R-tau-Protein) im Vergleich zu Gesunden vor. 
d) Eine ASO-Strategie bei Alzheimer Demenz liegt im Skipping von Exon 10 des MAPTGens.

e) Die proteolytische Spaltung von APP durch alpha-und dann beta-Sekretasen führt nicht zur Bildung von Abeta-Fragmenten.

\section{Welche Aussage ist falsch?}

a) Eine Veränderung der Expression von BDNF durch ASO-Strategien konnte bereits erfolgreich im Tiermodell gezeigt werden.

b) Im Tiermodell konnte gezeigt werden, dass die längerfristige Überexpression von BDNF mit schlechteren Lernleistungen und Gedächtnisfunktionen einherging. c) Die Reduktion der Neuropeptid-Y1-Rezeptorexpression durch ASO war im Tierversuch mit mehr Angstsymptome begleitet.

d) Durch ASOs konnte im Tiermodell eine verminderte Expression von striatalen Dopamin (D2)-Rezeptoren erzielt werden.

e) Ein ASO zur Behandlung von AutismusSpektrum-Störungen befindet sich bereits in klinischen Studien.

10. Welche Aussage zu den gesundheitspolitischen Implikationen von ASOs ist richtig?

a) Aufgrund der aktuellen Preise der ASOs muss eine komplexe Diskussion in der Gesellschaft zum ethisch gebotenen Behandlungsimperativ gegenüber der gesamtge- sellschaftlichen Leistungsfähigkeit geführt werden.

b) Für die zugelassenen Substanzen ist das Kosten-Nutzen-Verhältnis so positiv, dass für die Kostenträger kein Problem besteht.

c) Die Indikationen für den Einsatz von ASOs beziehen sich überwiegend auf sehr seltene Erkrankungen, so dass keine relevanten gesundheitspolitischen Fragen aufkommen.

d) Das Gesundheitssystem in Deutschland könnte problemlos auch wirksame ASOs für häufige Erkrankungen wie Alzheimer-Demenz finanzieren.

e) Die WHO diskutiert eine Zwangssteuer auf ASOs in Industrienationen zur Finanzierung der ASO-Verfügbarkeit in wirtschaftlich schwächeren Ländern. 